\title{
The regulation of MMP targeting to invadopodia during cancer metastasis
}

\author{
Abitha Jacob and Rytis Prekeris* \\ Department of Cell and Developmental Biology, School of Medicine, Anschutz Medical Campus, University of Colorado Denver, Aurora, CO, USA
}

\author{
Edited by: \\ Irina Kaverina, Vanderbilt University \\ Medical Center, USA

\section{Reviewed by:} \\ Frederique Gaits-lacovoni, Institut \\ National de la Santé et de la \\ Recherche Médicale U1048, France \\ Sébastien Roger, University of \\ Tours, France \\ *Correspondence: \\ Rytis Prekeris, Department of Cell \\ and Developmental Biology, School \\ of Medicine, Anschutz Medical \\ Campus, University of Colorado \\ Denver, 12801 East 17th Avenue, \\ Bldg. RC-1, Room L18-12402, \\ Aurora, CO, USA \\ e-mail:Rytis.Prekeris@ucdenver.edu
}

The dissemination of cancer cells from the primary tumor to a distant site, known as metastasis, is the main cause of mortality in cancer patients. Metastasis is a very complex cellular process that involves many steps, including the breaching of the basement membrane (BM) to allow the movement of cells through tissues. The BM breach occurs via highly regulated and localized remodeling of the extracellular matrix (ECM), which is mediated by formation of structures, known as invadopodia, and targeted secretion of matrix metalloproteinases (MMPs). Recently, invadopodia have emerged as key cellular structures that regulate the metastasis of many cancers. Furthermore, targeting of various cytoskeletal modulators and MMPs has been shown to play a major role in regulating invadopodia function. Here, we highlight recent findings regarding the regulation of protein targeting during invadopodia formation and function.

Keywords: MMP9, MMP2, Rab40B, membrane traffic, invadopodia, cancer, metastasis

\section{INTRODUCTION}

Although epithelial cancers are one of the leading causes of death, the mechanisms regulating the development and metastasis of carcinomas are not fully understood. Multiple studies suggest that the progression of tumors is dependent on the intrinsic properties of cancer cells, such as their ability to migrate and invade. Furthermore, many extrinsic factors, such as extracellular matrix (ECM) proteins, are also crucial for regulation of cancer metastasis. The ECM proteins that make up the specialized basement membrane (BM) serve as a barrier for cell invasion. However, the BM which is rich in laminin and collagen IV, also provides the substrate for adhesion of the migrating tumor cells. Furthermore, BM degradation results in the release/activation of various growth factors required for angiogenesis, tumor growth, and metastasis (Kalluri, 2003; Yurchenco, 2011). ECM degrading enzymes known as matrix metalloproteinases (MMPs) are known to play important roles in the degradation of the BM. Since several excellent reviews have already described the importance of MMPs in cancer cell growth and metastasis (Egeblad and Werb, 2002; Deryugina and Quigley, 2006; Fingleton, 2006; Gialeli et al., 2011), this review will focus on the mechanisms governing the targeting of MMPs to invadopodia.

\section{THE ROLE OF THE FAMILY OF MATRIX METALLOPROTEINASES (MMPS) IN CANCER CELL METASTASIS}

BM disruption involves a localized degradation of the ECM via the secretion of MMPs (Chamber and Matrisian, 1997). MMPs are a family of zinc endopeptidases that cleave ECM molecules and are subdivided into categories depending on their substrate specificity. The MMP family of enzymes includes not only the classical secreted and membrane bound MMPs, but also ADAMs (a disintegrin and metalloproteinase). ADAM metalloproteinases, also known as sheddases, are involved in cleaving various growth factors, cytokines, receptors, and adhesion molecules and are fundamental to development and homeostasis (Edwards et al., 2008). Like ADAMs, MMPs are also required for normal processes like tissue remodeling in embryonic development, wound healing, involution of mammary glands, angiogenesis, and ossification (Woessner, 1991). However, high levels of MMPs or aberrant MMP expression have often been correlated with pathological conditions like periodontitis, arthritis (Woessner, 1991) and have been implicated in multiple stages of cancer progression including invasion and metastasis (Egeblad and Werb, 2002). In this review, we will focus on the canonical MMPs, more specifically the ones that are targeted to the invadopodia and implicated in BM remodeling during metastasis of epithelial cancers. The association of MMPs with malignancies has been well documented with the majority of the evidence derived from mouse model studies and analysis of human patient samples. Based on substrate recognition, MMPs are categorized into interstitial collagenases, gelatinases, stromelysins, and membrane bound MMPs. Out of the 28 known MMPs, 14 have been implicated in cancer development and progression (Kohrmann et al., 2009). It has been shown that elevated expression of MMP1, 2, 3, 7, 9, 13, and 14 is positively correlated with tumor progression, metastasis, and poor overall prognosis (Lochter et al., 1998; Kerkela and Saarialho-Kere, 2003; Mook et al., 2004; Wagenaar-Miller et al., 2004; Ala-aho and Kahari, 2005; Bjorklund and Koivunen, 2005; Hofmann et al., 2005). Recently, it was shown that MMP9 drives tumor progression and metastasis of triple negative breast cancer (Mehner et al., 2014) and increased expression of MMP9 has been 
found in the early steps of melanoma (van den Oord et al., 1997). Expression of MMP9 has been associated both positively and negatively with survival rates in breast and colon cancer patients (Zeng et al., 1996; Takeha et al., 1997; Pacheco et al., 1998; Scorilas et al., 2001). Additionally, cancer cells have lesser capability to colonize the lungs of MMP2 or MMP9 deficient mice compared to wild type mice (Itoh et al., 1998, 1999) and cancer cell proliferation is decreased in tumors obtained from MMP9 knock-out mice (Bergers et al., 2000; Coussens et al., 2000). Overexpression of MMP3 and MMP14 (also known as MT1-MMP) has been shown to promote mammary carcinogenesis (Sternlicht et al., 1999; Ha et al., 2001). MMP12 expression in colon cancer has been correlated with increased survival (Yang et al., 2001). These studies indicate that several MMPs play a key role in cancer growth and metastasis. However, the expression levels and functions of individual MMPs are clearly dependent on the stage and type of cancer.

While MMP expression is increased in many cancers, the levels of activated rather than total MMPs appear to be a better indicator of tumor metastatic potential. There are two main mechanisms of post transcriptional regulation of MMP activity: activation of the latent precursor form (zymogen) and inhibition of the active enzyme by tissue inhibitors of MMPs or tissue inhibitors of metalloproteinases (TIMPs) (Polette et al., 2004). Most MMPs are secreted in an inactive pro-enzyme form and are activated extra-cellularly. An interesting property of MMPs is that they are capable of mutual activation. For example, MMP1 and MMP14 can activate MMP2 (Murphy and Crabbe, 1995; Strongin et al., 1995; Sang et al., 1996). The proteolytic activity of MMPs is also regulated by TIMPs, by binding to the zinc ion in their catalytic site (Gomis-Ruth et al., 1997; Fernandez-Catalan et al., 1998). There are four known TIMPs, of which TIMP1 and TIMP2 are the most promiscuous and inhibit the majority of MMPs. In vivo studies in mice have shown that overexpression of TIMP1 decreases metastasis to the brain and to the liver (Soloway et al., 1996; Kruger et al., 1997, 1998; Sternlicht and Werb, 2001). Overall, MMP activity is tightly regulated by different mechanisms and is involved in both normal and pathologic processes (Polette et al., 2004).

\section{THE ROLE OF INVADOPODIA FORMATION DURING CANCER CELL INVASION}

While the mechanisms mediating the movement of cells through the ECM remain to be fully characterized, it is now widely accepted that the formation of actin rich invasive protrusions is a key step during cancer cell invasion. These structures were identified in tissue culture cells and have been termed as podosomes or invadopodia (Tarone et al., 1985; Chen, 1989). While the functional differences between podosomes and invadopodia remain to be clearly defined, recent nomenclature has tried to distinguish podosomes as present in normal cells and invadopodia as present in cancer cells (Murphy and Courtneidge, 2011; Hoshino et al., 2013). Nevertheless, there are more similarities than differences between podosomes and invadopodia. Both these structures are actin rich and possess the ability to degrade the ECM (Linder and Kopp, 2005) However, they differ in their size, number, lifetime and location, which allows for differentiation between them during visualization (Linder and Kopp, 2005). Both podosomes and invadopodia are usually visualized with phalloidin, which stains filamentous actin and appears as punctate spots located below the nucleus. Podosomes have been observed in cells of monocytic lineage such as macrophages (Lehto et al., 1982; Linder et al., 1999) osteoclasts (Marchisio et al., 1984) and in induced smooth muscle cells (Gimona et al., 2003) as well as endothelial cells (Moreau et al., 2003; Osiak et al., 2005). In contrast, invadopodia are found in cells transformed with oncogenes (David-Pfeuty and Singer, 1980; Tarone et al., 1985) and are thought to protrude further into the matrix and invade more aggressively than podosomes (Weaver, 2008; Linder et al., 2011; Murphy and Courtneidge, 2011). A variety of actin regulators, scaffold proteins, small GTPases, and proteinases have been shown to play important roles in several steps of invadopodia formation. Several studies using animal xenografts and primary tumor cells from patients have also demonstrated the formation of invadopodia in vivo. Additionally, invadopodia has been observed in bladder cancer (Sutoh et al., 2010; Yamamoto et al., 2011), colorectal cancer (Schoumacher et al., 2010), breast cancer (Coopman et al., 1998; Yamaguchi et al., 2005a, 2011) squamous cell carcinoma (Takkunen et al., 2010) and glioblastoma (Stylli et al., 2008).

The ability of invadopodia to degrade ECM is attributed to the presence of matrix degrading enzymes such as MMPs. While the cellular machinery mediating the targeted release of MMPs from invadopodia remains to be defined, it is becoming clear that some MMPs are targeted and released from invadopodia to facilitate invasion (Nakahara et al., 1997). MMP14, MMP2, and MMP9 have all been shown to be important in cancer progression and enriched at the invadopodia (Monsky et al., 1993; Nakahara et al., 1997; Bourguignon et al., 1998; Artym et al., 2006; Clark and Weaver, 2008; Poincloux et al., 2009). MMP2 and MMP9 contain fibronectin repeats that help them recognize gelatin (denatured collagen) as a substrate (Polette et al., 2004) and Type IV collagen is the main constituent of the BM, one of the first barriers that cancer cells need to traverse to metastasize. In addition, MMP14 can recognize and cleave a broad spectrum of ECM substrates and also functions as an activator of MMP2 (Lebeau et al., 1999). Thus, the combined activity of MMP2, MMP9, and MMP14 is suggested to be an important step in initiating localized degradation of the BM during epithelial cancer metastasis (Nakahara et al., 1997; Chen and Wang, 1999). Even though this review focuses on the proteolytic aspect of MMP14, it is interesting to note that it can also function through a nonproteolytic mechanism. MMP14 can stimulate ATP production by activating Hypoxia- Inducible Factor-1(HIF-1) (Sakamoto and Seiki, 2010). The non-proteolytic activity of MMP14 also includes binding of its transmembrane domain to integrin $\beta 1$, which leads to MAPK activation, thereby regulating branching in mammary epithelium (Mori et al., 2013).

Invadopodia formation and function are complex cellular events that involve substantial reorganization in cytoskeleton dynamics and membrane transport. Recent studies have attempted to define different stages of invadopodia formation and function by using various criteria, including the recruitment of 
actin, targeted release of MMPs and the localized degradation of the ECM. Based on these, invadopodia formation has been divided into three stages, namely, initiation, assembly, and maturation. The following sections describe the stages of invadopodia formation in detail.

\section{INITIATION}

In the initiation phase of invadopodia formation, invadopodial precursors, or "buds" form at the cell periphery which are usually marked by actin puncta (Yamaguchi et al., 2005b). The process of invadopodia formation is initiated by growth factors such as epidermal growth factor (EGF), platelet derived growth factor (PDGF) and transforming growth factor- $\beta$ (TGF $\beta$ ). Growth factor receptor signaling activates Phosphatidylinositide 3-Kinase (P13K) leading to Src activation, which in turn phosphorylates multiple proteins including Tks5 (Tyrosine kinase substrate). Since the PX domain (phospholipid binding domain) of Tks5 has been shown to bind to PI3P and $\mathrm{PI}(3,4) \mathrm{P}_{2}$ (Abram et al., 2003), it was suggested that Tks5 localizes to $\mathrm{PI}(3,4) \mathrm{P}_{2}$ enriched regions of the plasma membrane, thus targeting Tks5 to initiate the invadopodia "bud" (Courtneidge et al., 2005). Src phosphorylates synaptojanin 2 to activate its phosphatase activity, which dephosphorylates $\mathrm{PI}(3,4,5) \mathrm{P}_{3}$ at the plasma membrane to form $\mathrm{PI}(3,4) \mathrm{P}_{2}$, thus forming the site for invadopodia formation (Chuang et al., 2012). Src mediated activation of the Abl-family kinase Arg also leads to the phosphorylation of cortactin, resulting in the recruitment of Nck1 to the invadopodia (Oser et al., 2009; Mader et al., 2011). Nck1 then recruits the Neural Wiskott-Aldrich syndrome protein (N-Wasp) complex to the invadopodia leading to Cdc42-dependent activation of $\mathrm{N}$ Wasp. N-Wasp in turn induces actin polymerization through the Arp2/3 complex, resulting in formation of invadopodial precursors (Yamaguchi et al., 2005a). The co-localization of cortactin with Tks5 in invadopodial "buds," led to the hypothesis that Tks5 acts a scaffolding protein that recruits the other cellular components required for initiation of invadopodia formation. However, some recent evidence suggests that Tks5 might instead be involved in invadopodia maturation (Sharma et al., 2013).

\section{ASSEMBLY}

Invadopodia are highly dynamic and motile structures that have been divided into two types based on their motility and lifetimes, motile short-lived and stationary long-lived invadopodia (Yamaguchi et al., 2005a). The short-lived invadopodia are thought to be precursors of fully functional mature invadopodia and could also be equivalent to podosomes (Yamaguchi et al., 2005a). In vitro, newly formed or early invadopodia can move laterally within the plasma membrane that faces the ECM. These motile invadopodia are then anchored and stabilized by actin polymerization and extension of the invadopodia (Yamaguchi et al., 2005a). A plethora of proteins are recruited to the invadopodial "bud" converting it from motile to stationary invadopodia. The precise order of how proteins are recruited is still unknown. Since Tks5 has five tandem SH3 domains, it is thought that Tks5 can scaffold several proteins like Nck1, Nck2, Grb2 (Growth factor receptor bound protein 2) and N-Wasp. It has also been proposed that Tks5, along with cortactin, recruits various actin regulators leading to nucleation of branched actin filaments and the formation of a stable actin core in the invadopodia (Clark and Weaver, 2008; Oser et al., 2009). Consistent with this hypothesis, it has been shown that phosphorylation of cortactin leads to dissociation of the cortactin/cofilin complex, which is an essential step in invadopodia formation and elongation (Oser et al., 2009). The dissociation of the cortactin/cofilin complex and the polymerization of actin is also a $\mathrm{pH}$-dependent process (Pope et al., 2004; Frantz et al., 2008).

\section{MATURATION}

Actin polymerization is crucial for formation and function of invadopodia. Inhibition of cofilin results in the formation of short-lived unstable invadopodia, which suggests that actin polymerization caused by cofilin is required for elongation and maturation of invadopodia (Yamaguchi et al., 2005a). Apart from the branched actin network, invadopodia also contain linear actin bundles (Li et al., 2010; Schoumacher et al., 2010). mDia2, a formin that induces the formation of linear actin networks, has been found to promote elongation and stability of invadopodia (Lizarraga et al., 2009). Fascin, another actin bundling protein has also been shown to promote elongation, stability and matrix degradation in invadopodia (Li et al., 2010; Schoumacher et al., 2010).

Src kinase is a major regulator of invadopodia formation and function. Interestingly, several other protein kinases including Abl kinases like Arg (Abl related gene) kinase have recently emerged as important players in invadopodia formation and maturation (Beaty et al., 2013). It was shown that $\beta 1$ integrin interacts with Arg leading to stimulation of Arg mediated cortactin phosphorylation, a key switch in promoting invadopodial maturation (Beaty et al., 2013). Additionally, $\beta 1$ integrin has been shown to localize to invadopodia and promote degradation of collagen type IV, the main constituent of the BM (Sameni et al., 2008), presumably by recruiting and docking proteases at the invadopodia. Separase, a gelatinolytic enzyme that has been shown to be enriched at the invadopodia, binds to $\alpha 3 \beta 1$ integrin resulting in the formation of functional invadopodia (Mueller et al., 1999). The ultrastructure of mature invadopodia has shown the presence of microtubules and many vesicles/endosomes indicative of active trafficking and a possible route for delivery of specific proteins like MMPs (Schoumacher et al., 2010). The activity of proteases docked at the invadopodia has been shown to be pH-dependent (Greco et al., 2014). Furthermore, it was demonstrated that the acidification of the peri-invadopodial space by the $\mathrm{Na}^{+} / \mathrm{H}^{+}$exchanger (NHE1) promotes ECM proteolysis (Busco et al., 2010).

\section{MECHANISMS MEDIATING MMP TARGETING TO INVADOPODIA}

The final maturation stage of the invadopodia involves targeted delivery and exocytosis of MMP2, MMP9, and MMP14. The appearance of these MMPs is generally considered to be a mark of functional mature invadopodia. As the result, much effort has been invested in understanding the regulation of MMP targeting to invadopodia, leading to recent studies defining the machinery governing MMP transport during cancer cell invasion. 

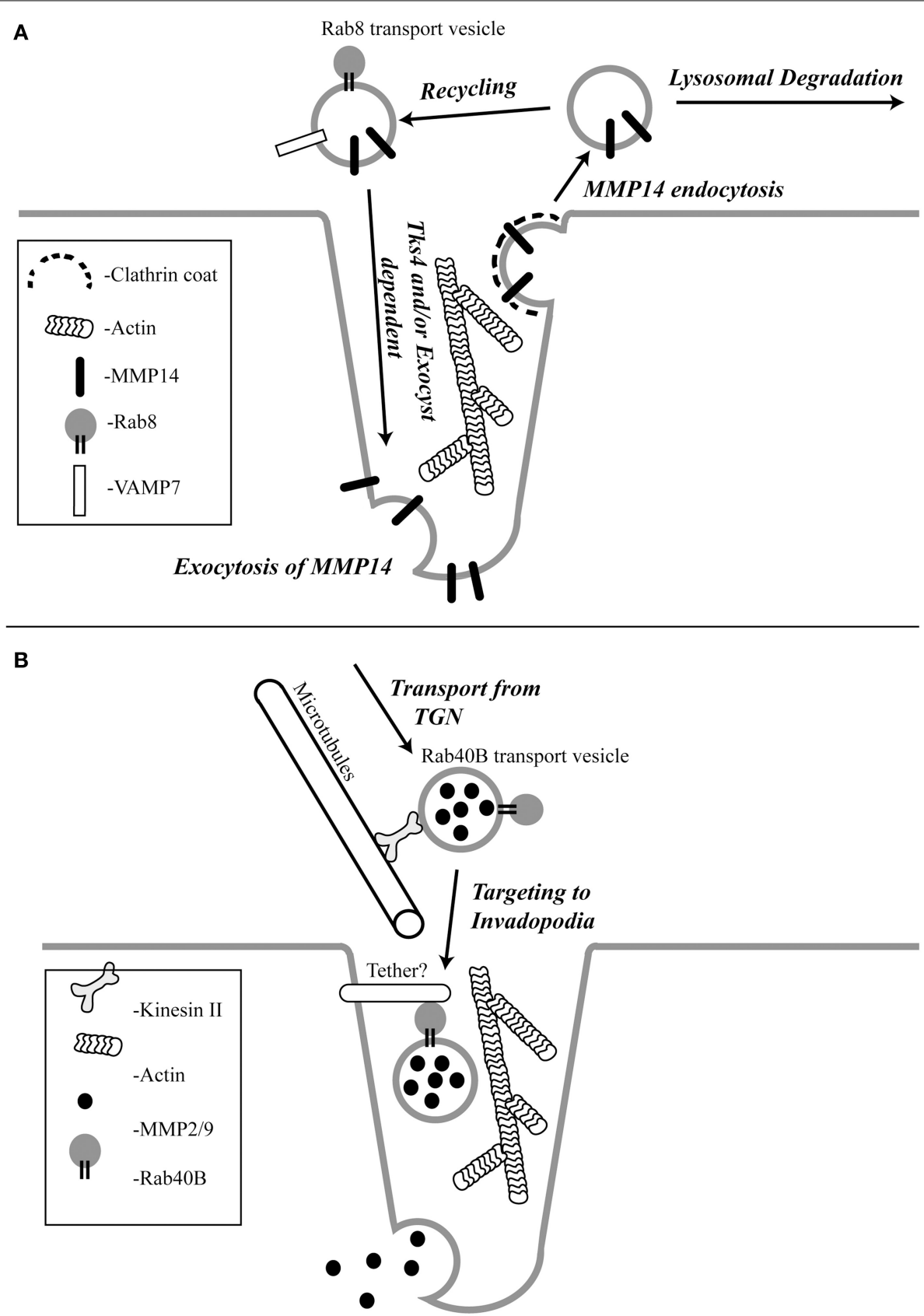

Exocytosis of MMP2/9

FIGURE 1 | The schematic representation of the pathways regulating MMP14 (A) and MMP2/9 (B) targeting to the invadopodia.

\section{TARGETING OF MMP14}

MMP14 is a membrane embedded MMP whose extracellular proteolytic activity is regulated by a balance between exocytosis and internalization via clathrin and/or caveolar mediated endocytosis (Remacle et al., 2003; Figure 1A). Once internalized, MMP14 is then either targeted to lysosomes for degradation (Jiang et al., 2001; Remacle et al., 2003) or shunted to endocytic recycling pathways, thus controlling the levels of active enzyme at the cell surface (Remacle et al., 2003; Figure 1A).
However, invasive cancer cells have mechanisms to counteract the removal of the active enzyme from the plasma membrane. Consistently, enrichment of active MMP14 at the invadopodia associated plasma membrane of tumor cells has been demonstrated in vitro (Nakahara et al., 1997; Artym et al., 2006; Clark and Weaver, 2008; Steffen et al., 2008). Endocytic recycling (Poincloux et al., 2009) exocytosis (Monteiro et al., 2013), Rab 8 (Bravo-Cordero et al., 2007) and Tks4 (Buschman et al., 2009) have all been shown to be involved in the localization of MMP14 
to the invadopodia. However, the exact mechanism governing MMP14 targeting remains to be fully understood. Rab8 GTPase has been shown to be involved in mobilization of MMP14 from intracellular storage compartments allowing polarized recruitment of MMP14 to the invasive front of cells (Bravo-Cordero et al., 2007). Some of the exocytic components shown to regulate delivery of MMP14 to the invadopodia are cortactin, the Exocyst complex (consists of 8 subunits) and VAMP7. Cortactin has been shown to regulate the cell surface expression of MMP14 (Clark et al., 2007). Sec8, a subunit of the Exocyst complex, was shown to localize at the invadopodia and is required for MMP14 targeting to the invadopodia (Monteiro et al., 2013; Figure 1A). Active RhoA and Cdc42 trigger the interaction between the Exocyst subunits Sec3 and Sec8 and the polarity protein IQGAP1. This interaction has been shown to be required for the accumulation of MMP14 at the invadopodia (Sakurai-Yageta et al., 2008). The Exocyst complex has also been shown to interact with Arp2/3 activator Wiskott-Aldrich syndrome protein and Scar homolog (WASH) to ensure focal delivery of MMP14 to the invadopodia (Monteiro et al., 2013). Since exocytosis depends on SNAREs (soluble N-ethylmaleimide sensitive factor attachment protein receptors) which drive the fusion of transport vesicles with the plasma membrane, several recent studies have investigated the role of SNAREs in mediating MMP14 transport. Consequently, it was shown that VAMP7 (vesicle associated membrane protein 7 ) is required for trafficking of MMP14 to invadopodia (Steffen et al., 2008; Figure 1A).

Transport vesicle targeting and fusion with its destination membranes often relies on specific tethering factors that impart specificity to membrane transport. The tethering factors regulating MMP14 targeting remain to be identified. However, Tks4, a scaffolding factor related to Tks5, has been shown to be required for the formation and function of invadopodia (Buschman et al., 2009). In the absence of Tks4, recruitment of MMP14 to podosomes is inhibited, implicating the role of Tks4 in targeting of MMP14 to the invadopodia (Buschman et al., 2009; Figure 1A). Additionally, cortactin was reported to have a novel role in invadopodial maturation and invasion by regulating secretion of MMP14 at the invadopodia (Clark and Weaver, 2008). However, it remains to be tested whether Tks4 and cortactin function as MMP14-vesicle tethers, or whether they affect MMP14 targeting indirectly by regulating the actin cytoskeleton within during invadopodia formation and maturation. Recently, it was also shown that Orail calcium channels can also regulate MMP14 targeting, since Oraill-mediated $\mathrm{Ca}^{2+}$ oscillations regulate MMP14 recycling to the plasma membrane (Sun et al., 2014).

\section{TARGETING OF MMP2 AND MMP9}

MMP2 and MMP9 are gelatinases that possess fibronectin type II repeats that allow them to degrade collagen and gelatin. Gelatinolytic degradation can cause the release of signaling molecules from the ECM that aid cell migration and angiogenesis. A lot of effort has been focused on understanding the transport and targeting of gelatinases because they are overexpressed in a variety of tumors and are associated with tumor aggressiveness and poor patient prognosis (Pacheco et al., 1998; Egeblad and Werb, 2002; Hiratsuka et al., 2002; van 't Veer et al., 2002). Although MMP2 and MMP9 have been shown to be enriched at the invadopodia, there is not much known about how these proteases are transported and targeted to the invadopodia. It has been reported that MMP2 and MMP9 are stored and transported in small vesicles that move along microtubules powered by kinesin in human melanoma cells (Schnaeker et al., 2004; Figure 1B). Similar to MMP14, the secretion of MMP2 and MMP9 were also shown to depend on cortactin (Clark and Weaver, 2008). Interestingly, in contrast to MMP14, endocytic transport and the Exocyst complex do not appear to play a role in targeted transport of MMP2 and MMP9 (Jacob et al., 2013). Instead, MMP2 and MMP9 are transported via specialized secretory vesicles directly from the trans-Golgi Network (TGN) to the invadopodia (Jacob et al., 2013). While much of the machinery mediating the formation and transport of these secretory vesicles remains to be defined, it was recently shown that Rab40b GTPase plays an important role in targeting MMP2/9 to the invadopodia (Jacob et al., 2013; Figure 1B).

\section{INVADOPODIA FORMATION AND FUNCTION IN VIVO}

Although there is an increasing amount of evidence demonstrating the existence of invadopodia in vitro, the formation and function of invadopodia in vivo is less well understood due to challenges associated with visualizing and distinguishing these structures in animal models. Cancer invasion usually occurs deep in tissues and these events are highly dynamic and unpredictable making it difficult to visualize invadopodia during primary tumor metastasis. Though the majority of invadopodial studies have been conducted in 2D tissue culture systems, some groups have studied invadopodia formation in 3D matrices as they better simulate the physiological in vivo environment. Such studies of invadopodia in complex 3D matrices have shown that the matrix degrading activity is localized to the base rather than the tip of the invadopodia (Wolf et al., 2007; Tolde et al., 2010). These 3D studies have also helped establish criteria for the identification of invadopodia in vivo and provide a good model to study formation of invadopodia.

Despite the challenges mentioned above, there is some compelling evidence drawn from elegant in vivo experiments that confirm that invadopodia are not just in vitro artifacts. Recently, the chorioallantoic membrane of the chicken embryo was used to visualize the intravascular formation of invadopodia and the extravasation of tumor cells into the stroma (Leong et al., 2014). The same group also showed that knocking down invadopodial components like cortactin, Tks4 and Tks5 decreases extravasation of cells into the lung stroma in tail vein injected mice. Intravital live animal imaging has also allowed the visualization of MtLn3-GFP (a highly invasive rat mammary carcinoma line) invading into blood vessels using protrusions identified as invadopodia-like structures using invadopodia markers such as cortactin and N-WASP (Lohmer et al., 2014). Using live-cell imaging, it was shown that during the uterine-vulval development in Caenorhabditis elegans, the anchor cell breaches the uterine and vulval basement membranes by making an invadopodium (Hagedorn et al., 2013). The Src-Tks5 pathway was shown to be required for the migration of neural crest cells using actin-rich 
protrusions in zebrafish (Murphy et al., 2011). Finally, it has also been shown that the intestinal epithelium of the zebrafish mutant meltdown forms invadopodia-like protrusions that invade into the stromal tissue in response to cues from the surrounding smooth muscle layer (Seiler et al., 2012).

\section{CONCLUSIONS AND FUTURE DIRECTIONS}

Significant advances have been made in understanding the formation and function of invadopodia. However, there are still a lot more unknowns regarding this subcellular structure. While all of the above mentioned studies have helped to confirm the physiological role of invadopodia as a structure used by invasive cells to penetrate the basement membrane, more evidence is required to elucidate the functional role of invadopodia in vivo and understand how widespread the use of invadopodia by cells is. Many questions regarding the importance of invadopodia in cancer invasion and metastasis still exist. Future studies in the field of invadopodia will need to focus on detection of invadopodia in human cancer samples as well as to identify the role of invadopodia in the different steps of the metastatic cascade. The other areas that require focus are the identification of components specific to invadopodia that can be targeted and the development of compounds that can specifically inhibit invadopodia formation and function. These issues will need to be addressed before invadopodia can become a candidate for development of new cancer therapies.

\section{ACKNOWLEDGMENTS}

We are grateful to Drs. Traci Lyons and Paul Jedlicka for critical reading of the manuscript. We apologize to all colleagues whose work could not be cited due to space limitations. We also acknowledge the financial support by NIH (DK064380 to Rytis Prekeris), Cancer League of Colorado (to Rytis Prekeris) and Susan G. Komen for the Cure foundation (BCTR0706749 to Rytis Prekeris).

\section{REFERENCES}

Abram, C. L., Seals, D. F., Pass, I., Salinsky, D., Maurer, L., Roth, T. M., et al. (2003). The adaptor protein fish associates with members of the ADAMs family and localizes to podosomes of Src-transformed cells. J. Biol. Chem. 278, 16844-16851. doi: 10.1074/jbc.M300267200

Ala-aho, R., and Kahari, V. M. (2005). Collagenases in cancer. Biochimie 87, 273-286. doi: 10.1016/j.biochi.2004.12.009

Artym, V. V., Zhang, Y., Moiseiwitsch, F. S., Yamada, K. M., and Mueller, S. C. (2006). Dynamic interactions of cortactin and membrane type 1 matrix metalloproteinase at invadopodia:defining the stages of invadopodia formaton and function. Cancer Res. 66, 3034-3043. doi: 10.1158/0008-5472.CAN-05-2177

Beaty, B. T., Sharma, V. P., Bravo-Cordero, J. J., Simpson, M. A., Eddy, R. J., Koleske, A. J., et al. (2013). Betal integrin regulates Arg to promote invadopodial maturation and matrix degradation. Mol. Biol. Cell 24, 1661-1675, S1661-S1611. doi: 10.1091/mbc.E12-12-0908

Bergers, G., Brekken, R., McMahon, G., Vu, T. H., Itoh, T., Tamaki, K., et al. (2000). Matrix metalloproteinase- 9 triggers the angiogenic switch during carcinogenesis. Nat. Cell Biol. 2, 737-744. doi: 10.1038/35036374

Bjorklund, M., and Koivunen, E. (2005). Gelatinase-mediated migration and invasion of cancer cells. Biochim. Biophys. Acta 1755, 37-69. doi: 10.1016/j.bbcan.2005.03.001

Bourguignon, L. Y., Gunja-Smith, Z., and Iida, N. (1998). CD44v(3,8-10) is involved in cytoskelton-mediated tumor cell migration and matrix metalloproteinase MMP-9 association in breast cancer cells. J. Cell Physiol. 176, 206-215.
Bravo-Cordero, J. J., Marrero-Diaz, R., Megias, D., Genis, L., Garcia-Grande, A., Garcia, M. A., et al. (2007). MT1-MMP proinvasive activity is regulated by a novel Rab8-dependent exocytic pathway. EMBO J. 26, 1499-1510. doi: 10.1038/sj.emboj.7601606

Buschman, M. D., Bromann, P. A., Cejudo-Martin, P., Wen, F., Pass, I., and Courtneidge, S. A. (2009). The novel adaptor protein Tks4 (SH3PXD2B) is required for functional podosome formation. Mol. Biol. Cell 20, 1302-1311. doi: 10.1091/mbc.E08-09-0949

Busco, G., Cardone, R. A., Greco, M. R., Bellizzi, A., Colella, M., Antelmi, E., et al. (2010). NHE1 promotes invadopodial ECM proteolysis through acidification of the peri-invadopodial space. FASEB J. 24, 3903-3915. doi: 10.1096/fj.09-149518

Chamber, A. F., and Matrisian, L. M. (1997). Changing views of the role of matrix metalloproteinases in metastasis. J. Natl. Cancer Inst. 89, 1260-1270. doi: 10.1093/jnci/89.17.1260

Chen, W. T. (1989). Proteolytic activity of specialized surface protrusions formed at rosette contact sites of transformed cells. J. Exp. Zool. 251, 167-185. doi: $10.1002 /$ jez. 1402510206

Chen, W. T., and Wang, J. Y. (1999). Specialized surface protrusions of invasive cells, invadopodia and lamellipodia, have differential MT1-MMP, MMP-2, and TIMP-2 localization. Ann. N.Y. Acad. Sci. 878, 361-371. doi: 10.1111/j.17496632.1999.tb07695.x

Chuang, Y., Xu, X., Kwiatkowska, A., Tsapraillis, G., Hwang, H., Petritis, K., et al. (2012). Regulation of synaptojanin 25 '-phosphatase activity by Src. Cell Adh. Migr. 6, 518-525. doi: 10.4161/cam.22139

Clark, E. S., and Weaver, A. M. (2008). A new role for cortactin in invadopodia:regulation of protease secretion. Eur. J. Cell Biol. 87, 581-590. doi: 10.1016/j.ejcb.2008.01.008

Clark, E. S., Whigham, A. S., Yarbrough, W. G., and Weaver, A. M. (2007). Cortactin is an essential regulator of matrix metalloproteinase secretion and extracellular matrix degradation in invadopodia. Cancer Res. 67, 4227-4235. doi: 10.1158/0008-5472.CAN-06-3928

Coopman, P. J., Do, M. T., Thompson, E. W., and Mueller, S. C. (1998). Phagocytosis of cross-linked gelatin matrix by human breast carcinoma cells correlates with their invasive capacity. Clin. Cancer Res. 4, 507-515.

Courtneidge, S. A., Azucena, E. F., Pass, I., Seals, D. F., and Tesfay, L. (2005). Src substrate Tks5, podosomes (invadopodia), and cancer cell invasion. Cold Spring Harb. Symp. Quant. Biol. 70, 161-171. doi: 10.1101/sqb.2005.70.014

Coussens, L. M., Tinkle, C. L., Hanahan, D., and Werb, Z. (2000). MMP-9 supplied by bone marrow-derived cells contributes to skin carcinogenesis. Cell 103, 481-490. doi: 10.1016/S0092-8674(00)00139-2

David-Pfeuty, T., and Singer, S. J. (1980). Altered distributions of the cytoskeletal proteins vinculin and alpha-actinin in cultured fibroblasts transformed by Rous sarcoma virus. Proc. Natl. Acad. Sci. U.S.A. 77, 6687-6691. doi: 10.1073/pnas.77.11.6687

Deryugina, E. I., and Quigley, J. P. (2006). Matrix metalloproteinases and tumor metastasis. Cancer Metastasis Rev. 25, 9-34. doi: 10.1007/s10555-006-7886-9

Edwards, D. R., Handsley, M. M., and Pennington, C. J. (2008). The ADAM metalloproteinases. Mol. Aspects Med. 29, 258-289. doi: 10.1016/j.mam.2008. 08.001

Egeblad, M., and Werb, Z. (2002). New functions of the matrix metalloproteinases in cancer progression. Nat. Rev. Cancer 2, 161-174. doi: 10.1038/nrc745

Fernandez-Catalan, C., Bode, W., Huber, R., Turk, D., Calvete, J. J., Lichte, A., et al. (1998). Crystal structure of the complex formed by the membrane type 1-matrix metalloproteinase with the tissue inhibitor of metalloproteinases2, the soluble progelatinase A receptor. $Е M B O ~ J . ~ 17,5238-5248$. doi: $10.1093 / \mathrm{emboj} / 17.17 .5238$

Fingleton, B. (2006). Matrix metalloproteinases: roles in cancer and metastasis. Front. Biosci. 11, 479-491. doi: 10.2741/1811

Frantz, C., Barreiro, G., Dominguez, L., Chen, X., Eddy, R., Condeelis, J., et al. (2008). Cofilin is a pH sensor for actin free barbed end formation: role of phosphoinositide binding. J. Cell Biol. 183, 865-879. doi: 10.1083/jcb.200804161

Gialeli, C., Theocharis, A. D., and Karamanos, N. K. (2011). Roles of matrix metalloproteinases in cancer progression and their pharmacological targeting. FEBS J. 278, 16-27. doi: 10.1111/j.1742-4658.2010.07919.x

Gimona, M., Kaverina, I., Resch, G. P., Vignal, E., and Burgstaller, G. (2003). Calponin repeats regulate actin filament stability and formation of podosomes in smooth muscle cells. Mol. Biol. Cell 14, 2482-2491. doi: 10.1091/mbc.E0211-0743 
Gomis-Ruth, F. X., Maskos, K., Betz, M., Bergner, A., Huber, R., Suzuki, K., et al. (1997). Mechanism of inhibition of the human matrix metalloproteinase stromelysin-1 by TIMP-1. Nature 389, 77-81. doi: 10.1038/ 37995

Greco, M. R., Antelmi, E., Busco, G., Guerra, L., Rubino, R., Casavola, V., et al. (2014). Protease activity at invadopodial focal digestive areas is dependent on NHE1-driven acidic pHe. Oncol. Rep. 31, 940-946. doi: 10.3892/or.2013.2923

Ha, H. Y., Moon, H. B., Nam, M. S., Lee, J. W., Ryoo, Z. Y., Lee, T. H., et al. (2001). Overexpression of membrane-type matrix metalloproteinase-1 gene induces mammary gland abnormalities and adenocarcinoma in transgenic mice. Cancer Res. 61, 984-990.

Hagedorn, E. J., Ziel, J. W., Morrissey, M. A., Linden, L. M., Wang, Z., Chi, Q., et al. (2013). The netrin receptor DCC focuses invadopodia-driven basement membrane transmigration in vivo. J. Cell Biol. 201, 903-913. doi: $10.1083 /$ jcb. 201301091

Hiratsuka, S., Nakamura, K., Iwai, S., Murakami, M., Itoh, T., Kijima, H., et al. (2002). MMP9 induction by vascular endothelial growth factor receptor-1 is involved in lung-specific metastasis. Cancer Cell 2, 289-300. doi: 10.1016/S15356108(02)00153-8

Hofmann, U. B., Houben, R., Brocker, E. B., and Becker, J. C. (2005). Role of matrix metalloproteinases in melanoma cell invasion. Biochimie 87, 307-314. doi: 10.1016/j.biochi.2005.01.013

Hoshino, D., Branch, K. M., and Weaver, A. M. (2013). Signaling inputs to invadopodia and podosomes. J. Cell Sci. 126, 2979-2989. doi: 10.1242/jcs.079475

Itoh, T., Tanioka, M., Matsuda, H., Nishimoto, H., Yoshioka, T., Suzuki, R., et al. (1999). Experimental metastasis is suppressed in MMP-9-deficient mice. Clin. Exp. Metastasis 17, 177-181. doi: 10.1023/A:1006603723759

Itoh, T., Tanioka, M., Yoshida, H., Yoshioka, T., Nishimoto, H., and Itohara, S. (1998). Reduced angiogenesis and tumor progression in gelatinase A- deficient mice. Cancer Res. 58, 1048-1051.

Jacob, A., Jing, J., Lee, J., Schedin, P., Peden, A. A., Junutula, J. R., et al. (2013). Rab40b regulates MMP2 and MMP9 targeting to the invadopodia during breast cancer cell invasion. J. Cell Sci. 126, 4647-4658. doi: 10.1242/jcs. 126573

Jiang, A., Lehti, K., Wang, X., Weiss, S. J., Keski-Oja, J., and Pei, D. (2001). Regulation of membrane-type matrix metalloproteinase 1 activity by dynaminmediated endocytosis. Proc. Natl. Acad. Sci. U.S.A. 98, 13693-13698. doi: 10.1073/pnas. 241293698

Kalluri, R. (2003). Basement membranes: structure, assembly and role in tumour angiogenesis. Nat. Rev. Cancer 3, 422-433. doi: 10.1038/nrc1094

Kerkela, E., and Saarialho-Kere, U. (2003). Matrix metalloproteinases in tumor progression: focus on basal and squamous cell skin cancer. Exp. Dermatol. 12, 109-125. doi: 10.1034/j.1600-0625.2003.120201.x

Kohrmann, A., Kammerer, U., Kapp, M., Dietl, J., and Anacker, J. (2009). Expression of matrix metalloproteinases (MMPs) in primary human breast cancer and breast cancer cell lines: new findings and review of the literature. $B M C$ Cancer 9:188. doi: 10.1186/1471-2407-9-188

Kruger, A., Fata, J. E., and Khokha, R. (1997). Altered tumor growth and metastasis of a T-cell lymphoma in Timp-1 transgenic mice. Blood 90, 1993-2000.

Kruger, A., Sanchez-Sweatman, O. H., Martin, D. C., Fata, J. E., Ho, A. T., Orr, F. W., et al. (1998). Host TIMP-1 overexpression confers resistance to experimental brain metastasis of a fibrosarcoma cell line. Oncogene 16, 2419-2423. doi: 10.1038/sj.onc. 1201774

Lebeau, A., Nerlich, A. G., Sauer, U., Lichtinghagen, R., and Lohrs, U. (1999). Tissue distribution of major matrix metalloproteinases and their transcripts in human breast carcinomas. Anticancer Res. 19, 4257-4264.

Lehto, V. P., Hovi, T., Vartio, T., Badley, R. A., and Virtanen, I. (1982). Reorganization of cytoskeletal and contractile elements during transition of human monocytes into adherent macrophages. Lab. Invest. 47, 391-399.

Leong, H. S., Robertson, A. E., Stoletov, K., Leith, S. J., Chin, C. A., Chien, A. E., et al. (2014). Invadopodia are required for cancer cell extravasation and are a therapeutic target for metastasis. Cell Rep. 8, 1558-1570. doi: 10.1016/j.celrep.2014.07.050

Li, A., Dawson, J. C., Forero-Vargas, M., Spence, H. J., Yu, X., Konig, I., et al. (2010). The actin-bundling protein fascin stabilizes actin in invadopodia and potentiates protrusive invasion. Curr. Biol. 20, 339-345. doi: 10.1016/j.cub.2009. 12.035

Linder, S., and Kopp, P. (2005). Podosomes at a glance. J. Cell Sci. 118, 2079-2082. doi: $10.1242 /$ jcs. 02390
Linder, S., Nelson, D., Weiss, M., and Aepfelbacher, M. (1999). Wiskott-Aldrich syndrome protein regulates podosomes in primary human macrophages. Proc. Natl. Acad. Sci. U.S.A. 96, 9648-9653. doi: 10.1073/pnas.96.17.9648

Linder, S., Wiesner, C., and Himmel, M. (2011). Degrading devices: invadosomes in proteolytic cell invasion. Annu. Rev. Cell Dev. Biol. 27, 185-211. doi: 10.1146/annurev-cellbio-092910-154216

Lizarraga, F., Poincloux, R., Romao, M., Montagnac, G., Le Dez, G., Bonne, I., et al. (2009). Diaphanous-related formins are required for invadopodia formation and invasion of breast tumor cells. Cancer Res. 69, 2792-2800. doi: 10.1158/0008-5472.CAN-08-3709

Lochter, A., Sternlicht, M. D., Werb, Z., and Bissell, M. J. (1998). The significance of matrix metalloproteinases during early stages of tumor progression. Ann. N.Y. Acad. Sci. 857, 180-193. doi: 10.1111/j.1749-6632.1998.tb 10116.x

Lohmer, L. L., Kelley, L. C., Hagedorn, E. J., and Sherwood, D. R. (2014). Invadopodia and basement membrane invasion in vivo. Cell Adh. Migr. 8, 246-255. doi: 10.4161/cam.28406

Mader, C. C., Oser, M., Magalhaes, M. A., Bravo-Cordero, J. J., Condeelis, J., Koleske, A. J., et al. (2011). An EGFR-Src-Arg-cortactin pathway mediates functional maturation of invadopodia and breast cancer cell invasion. Cancer Res. 71, 1730-1741. doi: 10.1158/0008-5472.CAN-10-1432

Marchisio, P. C., Cirillo, D., Naldini, L., Primavera, M. V., Teti, A., and ZamboninZallone, A. (1984). Cell-substratum interaction of cultured avian osteoclasts is mediated by specific adhesion structures. J. Cell Biol. 99, 1696-1705. doi: 10.1083/jcb.99.5.1696

Mehner, C., Hockla, A., Miller, E., Ran, S., Radisky, D. C., and Radisky, E. S. (2014). Tumor cell-produced matrix metalloproteinase 9 (MMP-9) drives malignant progression and metastasis of basal-like triple negative breast cancer. Oncotarget 5, 2736-2749. doi: 10.1371/journal.pone.0013066

Monsky, W. L., Kelly, T., and Lin, C. Y. (1993). Binding and localization of M(r) 72,000 matrix metalloproteinase at cell surface invadopodia. Cancer Res. 53, 3159-3164.

Monteiro, P., Rosse, C., Castro-Castro, A., Irondelle, M., Lagoutte, E., PaulGilloteaux, P., et al. (2013). Endosomal WASH and exocyst complexes control exocytosis of MT1-MMP at invadopodia. J. Cell Biol. 203, 1063-1079. doi: $10.1083 /$ jcb. 201306162

Mook, O. R., Frederiks, W. M., and Van Noorden, C. J. (2004). The role of gelatinases in colorectal cancer progression and metastasis. Biochim. Biophys. Acta 1705, 69-89. doi: 10.1016/j.bbcan.2004.09.006

Moreau, V., Tatin, F., Varon, C., and Genot, E. (2003). Actin can reorganize into podosomes in aortic endothelial cells, a process controlled by Cdc42 and RhoA. Mol. Cell. Biol. 23, 6809-6822. doi: 10.1128/MCB.23.19.6809-6822.2003

Mori, H., Lo, A. T., Inman, J. L., Alcaraz, J., Ghajar, C. M., Mott, J. D., et al. (2013). Transmembrane/cytoplasmic, rather than catalytic, domains of Mmp14 signal to MAPK activation and mammary branching morphogenesis via binding to integrin betal. Development 140, 343-352. doi: 10.1242/dev.084236

Mueller, S. C., Ghersi, G., Akiyama, S. K., Sang, Q. X., Howard, L., Pineiro-Sanchez, M., et al. (1999). A novel protease-docking function of integrin at invadopodia. J. Biol. Chem. 274, 24947-24952. doi: 10.1074/jbc.274.35.24947

Murphy, D. A., and Courtneidge, S. A. (2011). The 'ins' and 'outs' of podosomes and invadopodia:characteristics, formation and function. Nat. Rev. Mol. Cell Biol. 12, 413-426. doi: 10.1038/nrm3141

Murphy, G., and Crabbe, T. (1995). Gelatinases A and B. Methods Enzymol. 248, 470-484. doi: 10.1016/0076-6879(95)48030-7

Murphy, D. A., Diaz, B., Bromann, P. A., Tsai, J. H., Kawakami, Y., Maurer, J., et al. (2011). A Src-Tks5 pathway is required for neural crest cell migration during embryonic development. PLoS ONE 6:e22499. doi: 10.1371/journal.pone.0022499

Nakahara, H., Howard, L., Thompson, E. W., Sato, H., Seiki, M., Yeh, Y., et al. (1997). Transmembrane/cytoplasmic domain-mediated membrane type1matrix metalloprotease docking to invadopodiais required for cell invasion. Proc. Natl. Acad. Sci. U.S.A. 94, 7959-7964. doi: 10.1073/pnas.94.15.7959

Oser, M., Yamaguchi, H., Mader, C. C., Bravo-Cordero, J. J., Arias, M., Chen, X., et al. (2009). Cortactin regulates cofilin and N-WASp activities to control the stages of invadopodium assembly and maturation. J. Cell Biol. 186, 571-587. doi: $10.1083 /$ jcb. 200812176

Osiak, A. E., Zenner, G., and Linder, S. (2005). Subconfluent endothelial cells form podosomes downstream of cytokine and RhoGTPase signaling. Exp. Cell Res. 307, 342-353. doi: 10.1016/j.yexcr.2005.03.035 
Pacheco, M. M., Mourao, M., Mantovani, E. B., Nishimoto, I. N., and Brentani, M. M. (1998). Expression of gelatinases A and B, stromelysin-3 and matrilysin genes in breast carcinomas: clinico-pathological correlations. Clin. Exp. Metastasis 16, 577-585. doi: 10.1023/A:1006580415796

Poincloux, R., Lizarraga, F., and Chavrier, P. (2009). Matrix invasion by tumor cells: a focus on MT1-MMP trafficking to invadopodia. J. Cell Sci. 122, 3015-3024. doi: $10.1242 /$ jcs.034561

Polette, M., Nawrocki-Raby, B., Gilles, C., Clavel, C., and Birembaut, P. (2004). Tumour invasion and matrix metalloproteinases. Crit. Rev. Oncol. Hematol. 49, 179-186. doi: 10.1016/j.critrevonc.2003.10.008

Pope, B. J., Zierler-Gould, K. M., Kuhne, R., Weeds, A. G., and Ball, L. J. (2004). Solution structure of human cofilin: actin binding, $\mathrm{pH}$ sensitivity, and relationship to actin-depolymerizing factor. J. Biol. Chem. 279, 4840-4848. doi: 10.1074/jbc.M310148200

Remacle, A., Murphy, G., and Roghi, C. (2003). Membrane type I-matrix metalloproteinase (MT1-MMP) is internalised by two different pathways and is recycled to the cell surface. J. Cell Sci. 116, 3905-3916. doi: 10.1242/jcs. 00710

Sakamoto, T., and Seiki, M. (2010). A membrane protease regulates energy production in macrophages by activating hypoxia-inducible factor-1 via a non-proteolytic mechanism. J. Biol. Chem. 285, 29951-29964. doi: 10.1074/jbc.M110.132704

Sakurai-Yageta, M., Recchi, C., Le Dez, G., Sibarita, J. B., Daviet, L., Camonis, J., et al. (2008). The interaction of IQGAP1 with the exocyst complex is required for tumor cell invasion downstream of Cdc42 and RhoA. J. Cell Biol. 181, 985-998. doi: 10.1083/jcb.200709076

Sameni, M., Dosescu, J., Yamada, K. M., Sloane, B. F., and Cavallo-Medved, D. (2008). Functional live-cell imaging demonstrates that betal-integrin promotes type IV collagen degradation by breast and prostate cancer cells. Mol. Imaging 7, 199-213.

Sang, Q. A., Bodden, M. K., and Windsor, L. J. (1996). Activation of human progelatinase A by collagenase and matrilysin: activation of procollagenase by matrilysin. J. Protein Chem. 15, 243-253. doi: 10.1007/BF018 87112

Schnaeker, E. M., Ossig, R., Ludwig, T., Dreier, R., Oberleithner, H., Wilhelmi, M., et al. (2004). Microtubule-dependent matrix metalloproteinase2/matrix metalloproteinase- 9 exocytosis: prerequisite in human melanoma cell invasion. Cancer Res. 64, 8924-8931. doi: 10.1158/0008-5472.CAN04-0324

Schoumacher, M., Goldman, R. D., Louvard, D., and Vignjevic, D. M. (2010). Actin, microtubules, and vimentin intermediate filaments cooperate for elongation of invadopodia. J. Cell Biol. 189, 541-556. doi: 10.1083/jcb.200909113

Scorilas, A., Karameris, A., Arnogiannaki, N., Ardavanis, A., Bassilopoulos, P., Trangas, T., et al. (2001). Overexpression of matrix-metalloproteinase- 9 in human breast cancer: a potential favourable indicator in node-negative patients. Br. J. Cancer 84, 1488-1496. doi: 10.1054/bjoc.2001.1810

Seiler, C., Davuluri, G., Abrams, J., Byfield, F. J., Janmey, P. A., and Pack, M. (2012). Smooth muscle tension induces invasive remodeling of the zebrafish intestine. PLoS Biol. 10:e1001386. doi: 10.1371/journal.pbio.1001386

Sharma, V. P., Eddy, R., Entenberg, D., Kai, M., Gertler, F. B., and Condeelis, J. (2013). Tks5 and SHIP2 regulate invadopodium maturation, but not initiation, in breast carcinoma cells. Curr. Biol. 23, 2079-2089. doi: 10.1016/j.cub.2013.08.044

Soloway, P. D., Alexander, C. M., Werb, Z., and Jaenisch, R. (1996). Targeted mutagenesis of Timp-1 reveals that lung tumor invasion is influenced by Timp-1 genotype of the tumor but not by that of the host. Oncogene 13, 2307-2314.

Steffen, A., Le Dez, G., Poincloux, R., Recchi, C., Nassoy, P., Rottner, K., et al. (2008). MT1-MMP-dependent invasion is regulated by TI-VAMP/VAMP7. Curr. Biol. 18, 926-931. doi: 10.1016/j.cub.2008.05.044

Sternlicht, M. D., and Werb, Z. (2001). How matrix metalloproteinases regulate cell behavior. Annu. Rev. Cell Dev. Biol. 17, 463-516. doi: 10.1146/annurev.cellbio.17.1.463

Sternlicht, M. D., Lochter, A., Sympson, C. J., Huey, B., Rougier, J. P., Gray, J. W., et al. (1999). The stromal proteinase MMP3/stromelysin-1 promotes mammary carcinogenesis. Cell 98, 137-146. doi: 10.1016/S0092-8674(00)81009-0

Strongin, A. Y., Collier, I., Bannikov, G., Marmer, B. L., Grant, G. A., and Goldberg, G. I. (1995). Mechanism of cell surface activation of 72-kDa type IV collagenase. Isolation of the activated form of the membrane metalloprotease. J. Biol. Chem. 270, 5331-5338. doi: 10.1074/jbc.270.10.5331
Stylli, S. S., Kaye, A. H., and Lock, P. (2008). Invadopodia: at the cutting edge of tumour invasion. J. Clin. Neurosci. 15, 725-737. doi: 10.1016/j.jocn.2008. 03.003

Sun, J., Lu, F., He, H., Shen, J., Messina, J., Mathew, R., et al. (2014). STIM1and Orail-mediated $\mathrm{Ca} 2+$ oscillation orchestrates invadopodium formation and melanoma invasion. J. Cell Biol. 207, 535-548. doi: 10.1083/jcb.2014 07082

Sutoh, M., Hashimoto, Y., Yoneyama, T., Yamamoto, H., Hatakeyama, S., Koie, T., et al. (2010). Invadopodia formation by bladder tumor cells. Oncol. Res. 19, 85-92. doi: 10.3727/096504010X12875107808008

Takeha, S., Fujiyama, Y., Bamba, T., Sorsa, T., Nagura, H., and Ohtani, H. (1997) Stromal expression of MMP-9 and urokinase receptor is inversely associated with liver metastasis and with infiltrating growth in human colorectal cancer: a novel approach from immune/inflammatory aspect. Jpn. J. Cancer Res. 88, 72-81. doi: 10.1111/j.1349-7006.1997.tb00304.x

Takkunen, M., Hukkanen, M., Liljestrom, M., Grenman, R., and Virtanen, I. (2010). Podosome-like structures of non-invasive carcinoma cells are replaced in epithelial-mesenchymal transition by actin comet-embedded invadopodia. J. Cell. Mol. Med. 14, 1569-1593. doi: 10.1111/j.1582-4934.2009. 00868.x

Tarone, G., Cirillo, D., Giancotti, F. G., Comoglio, P. M., and Marchisio, P. C. (1985). Rous sarcoma virus-transformed fibroblasts adhere primarily at discrete protrusions of the ventral membrane called podosomes. Exp. Cell Res. 159, 141-157. doi: 10.1016/S0014-4827(85)80044-6

Tolde, O., Rosel, D., Vesely, P., and Brabek, J. (2010). The structure of invadopodia in a complex 3D environment. Eur. J. Cell Biol. 89, 674-680. doi: 10.1016/j.ejcb.2010.04.003

van den Oord, J. J., Paemen, L., Opdenakker, G., and de Wolf-Peeters, C. (1997). Expression of gelatinase $\mathrm{B}$ and the extracellular matrix metalloproteinase inducer EMMPRIN in benign and malignant pigment cell lesions of the skin. Am. J. Pathol. 151, 665-670.

van 't Veer, L. J., Dai, H., van de Vijver, M. J., He, Y. D., Hart, A. A., Mao, M., et al. (2002). Gene expression profiling predicts clinical outcome of breast cancer. Nature 415, 530-536. doi: 10.1038/415530a

Wagenaar-Miller, R. A., Gorden, L., and Matrisian, L. M. (2004). Matrix metalloproteinases in colorectal cancer: is it worth talking about? Cancer Metastasis Rev. 23, 119-135. doi: 10.1023/A:1025819214508

Weaver, A. M. (2008). Invadopodia. Curr. Biol. 18, R362-R364. doi: 10.1016/j.cub. 2008.02.028

Woessner, J. F. Jr. (1991). Matrix metalloproteinases and their inhibitors in connective tissue remodeling. FASEB J. 5, 2145-2154.

Wolf, K., Wu, Y. I., Liu, Y., Geiger, J., Tam, E., Overall, C., et al. (2007), Multi-step pericellular proteolysis controls the transition from individual to collective cancer cell invasion. Nat. Cell Biol. 9, 893-904. doi: 10.1038/ ncb1616

Yamaguchi, H., Lorenz, M., Kempiak, S., Sarmiento, C., Coniglio, S., Symons, M., et al. (2005a). Molecular mechanisms of invadopodium formation: the role of the N-WASP-Arp2/3 complex pathway and cofilin. J. Cell Biol. 168, 441-452. doi: $10.1083 /$ jcb. 200407076

Yamaguchi, H., Wyckoff, J., and Condeelis, J. (2005b). Cell migration in tumors. Curr. Opin. Cell Biol. 17, 559-564. doi: 10.1016/j.ceb.2005.08.002

Yamaguchi, H., Yoshida, S., Muroi, E., Yoshida, N., Kawamura, M., Kouchi, Z., et al. (2011). Phosphoinositide 3-kinase signaling pathway mediated by p110alpha regulates invadopodia formation. J. Cell Biol. 193, 1275-1288. doi: 10.1083/jcb.201009126

Yamamoto, H., Sutoh, M., Hatakeyama, S., Hashimoto, Y., Yoneyama, T., Koie, T., et al. (2011). Requirement for FBP17 in invadopodia formation by invasive bladder tumor cells. J. Urol. 185, 1930-1938. doi: 10.1016/j.juro.2010. 12.027

Yang, W., Arii, S., Gorrin-Rivas, M. J., Mori, A., Onodera, H., and Imamura, M. (2001). Human macrophage metalloelastase gene expression in colorectal carcinoma and its clinicopathologic significance. Cancer 91, 1277-1283. doi: 10.1002/1097-0142(20010401)91:7<1277::AID-CNCR1129>3.0.CO;2-H

Yurchenco, P. D. (2011). Basement membranes: cell scaffoldings and signaling platforms. Cold Spring Harb. Perspect. Biol. 3:a004911. doi: 10.1101/cshperspect.a004911

Zeng, Z. S., Huang, Y., Cohen, A. M., and Guillem, J. G. (1996). Prediction of colorectal cancer relapse and survival via tissue RNA levels of matrix metalloproteinase-9. J. Clin. Oncol. 14, 3133-3140. 
Conflict of Interest Statement: The authors declare that the research was conducted in the absence of any commercial or financial relationships that could be construed as a potential conflict of interest.

Received: 25 November 2014; accepted: 09 January 2015; published online: 02 February 2015.

Citation: Jacob $A$ and Prekeris $R$ (2015) The regulation of MMP targeting to invadopodia during cancer metastasis. Front. Cell Dev. Biol. 3:4. doi: 10.3389/fcell. 2015.00004
This article was submitted to Membrane Traffic, a section of the journal Frontiers in Cell and Developmental Biology.

Copyright (c) 2015 Jacob and Prekeris. This is an open-access article distributed under the terms of the Creative Commons Attribution License (CC BY).

The use, distribution or reproduction in other forums is permitted, provided the original author(s) or licensor are credited and that the original publication in this journal is cited, in accordance with accepted academic practice. No use, distribution or reproduction is permitted which does not comply with these terms. 